\title{
Radio over Fiber Based Networks for the Smart Grid
}

\author{
A. Ghassemi \\ Dept. of Electrical Eng. \\ University of Tehran \\ Tehran, Iran \\ aghassem@ieee.org
}

\author{
T. A. Gulliver \\ Dept. of Electrical Eng. \\ University of Victoria \\ Victoria, BC Canada \\ agullive@ece.uvic.ca
}

\author{
J. M. Cioffi \\ Dept. of Electrical Eng. \\ Stanford University \\ Stanford, CA USA \\ cioffi@stanford.edu
}

\author{
G. K. Karagiannidis \\ Dept. of Electrical Eng. \\ Aristotle University of Thessaloniki \\ Thessaloniki, Greece \\ geokarag@auth.gr
}

\begin{abstract}
In this paper, the application of radio over fiber (RoF) based wireless communications for smart metering and power distribution is introduced. A RoF based network has the potential to enhance both the coverage and spectral efficiency of smart grid wireless networks since its major benefit is adaptability to temporal and spatial traffic variations. A clustered architecture is presented which provides signal diversity to enhance the coverage and capacity of smart grid communications. Examples are presented to illustrate the improvement in coverage and latency of a smart grid wireless network when RoF is used instead of a conventional wireless network.
\end{abstract}

\section{INTRODUCTION}

A key component of the smart grid is a flexible and intelligent communications infrastructure that provides utilities with remote access and control of the grid components, including real-time sensing and measurement [1]. Wireless technologies are very important role for smart grid communications because of their low installation costs, simple deployment, and ease of connection to provide access throughout the power grid. However, wireless networking in the smart grid context has several design requirements that differ from other applications.

Smart grid wireless networks include diverse grid components (e.g., smart meters and intelligent sensors), to support numerous applications such as demand response and distribution automation. Many smart devices may simultaneously transmit data (e.g., in response to a power outage), resulting in severe network congestion. This poses a major challenge in ensuring the reliability and timeliness of the data transmitted over these networks. The diversity and nature of some components (e.g., renewable energy resources), can create time-varying traffic while smart devices may also be sparsely or densely placed, and in close proximity to other grid devices. This causes spatially varying traffic demands with different coverage areas. Based on the above distinct challenges, advanced techniques (e.g., cognitive radio [2][3] and radio over fiber), must be developed to maximize the efficiency of smart grid wireless networks.

This paper presents the application of radio over fiber (RoF) distributed antenna systems in the smart grid. In particular, a RoF based system is considered to improve the efficiency and reliability of communications in the advanced metering infrastructure (AMI) and power distribution system. RoF interconnects spatially separated remote antenna units (RAUs) to centralized control units through an optical fiber link where radio components are co-located [4]. Thus, it integrates radio and optical fiber technologies to exploit their best features and provide efficient and effective network services. The optical side provides reliable transmission for smart meter and intelligent sensor data, while the wireless side allows flexible access to remote locations and broad coverage. The centralized architecture of RoF based networks facilitates radio resource allocation to improve coverage, support temporal traffic variations, and prevent network congestion.

A clustered architecture is first developed for efficient placement of RAUs to handle spatial traffic variations and improve coverage. Then the RAUs are dynamically activated to achieve transmission diversity gains and provide reliable communication links. Channel allocation via the proposed cluster architecture is introduced as a means of enhancing capacity for future smart grid traffic and applications. Deployment guidelines are developed for RoF based communications in the smart grid. The results presented indicate that significant coverage and latency improvements are possible with a RoF based smart grid network over a conventional wireless network. To the best of our knowledge, this is the first investigation of RoF in smart grid networks.

The remainder of this paper is organized as follows. Section II presents an overview of the smart grid wireless communications infrastructure, including the AMI. The main communications challenges are identified in the hierarchical architecture of smart grid wireless networks. Then the RoF based network is introduced and its benefits for smart grid communications are discussed. Numerical results are presented in Section III for a RoF network with clustered RAUs. Finally, some conclusions are given in Section IV.

\section{The Advanced Metering Infrastructure And SMART GRID WIRELESS COMMUNICATIONS ARCHiteCTURE}

This section provides an overview of the advanced metering infrastructure (AMI), with a focus on its major components and the related operation and management systems. The smart grid wireless network architecture is then described. The design challenges for smart metering and distribution 
communications networks are presented. Then benefits of RoF technology for smart grid wireless networks are discussed.

\section{A. Advanced Metering Infrastructure}

The advanced metering infrastructure (AMI), commonly known as smart metering, uses bidirectional communications to provide two major classes of services. First, it provides consumers with energy management (e.g., consumption data and outage reports) and other control information. It also enables utilities to collect power quality and load profile data. Second, it provides control information (e.g., alerts and equipment settings), for actuators/sensors located within the power distribution network to support advanced distribution automation. The AMI also collects data from actuators/sensors for power outage detection and other purposes. The major AMI components are the smart meters installed on the user side, and the metering data management systems (MDMSs) located in utility control centers [5].

A smart meter measures real-time energy consumption and transmits this data to a MDMS. The MDMS processes and manages data on energy consumption and fault detection. Analysis tools are used to facilitate the interaction with other operation and management systems. This includes the outage management system (OMS), the geographic information system (GIS), the consumer information system (CIS) that manages billing and customer information, and the distribution management system (DMS) that provides power quality management and power demand forecasting [5].

\section{B. The Smart Grid Wireless Network Topology}

To support the AMI and power distribution system, the smart grid wireless network has a hierarchical architecture that extends across multiple grid operational layers. It consists of home area networks (HANs), neighborhood area networks (NANs), and wide area networks (WANs) which have different coverage areas as shown in Fig. 1.

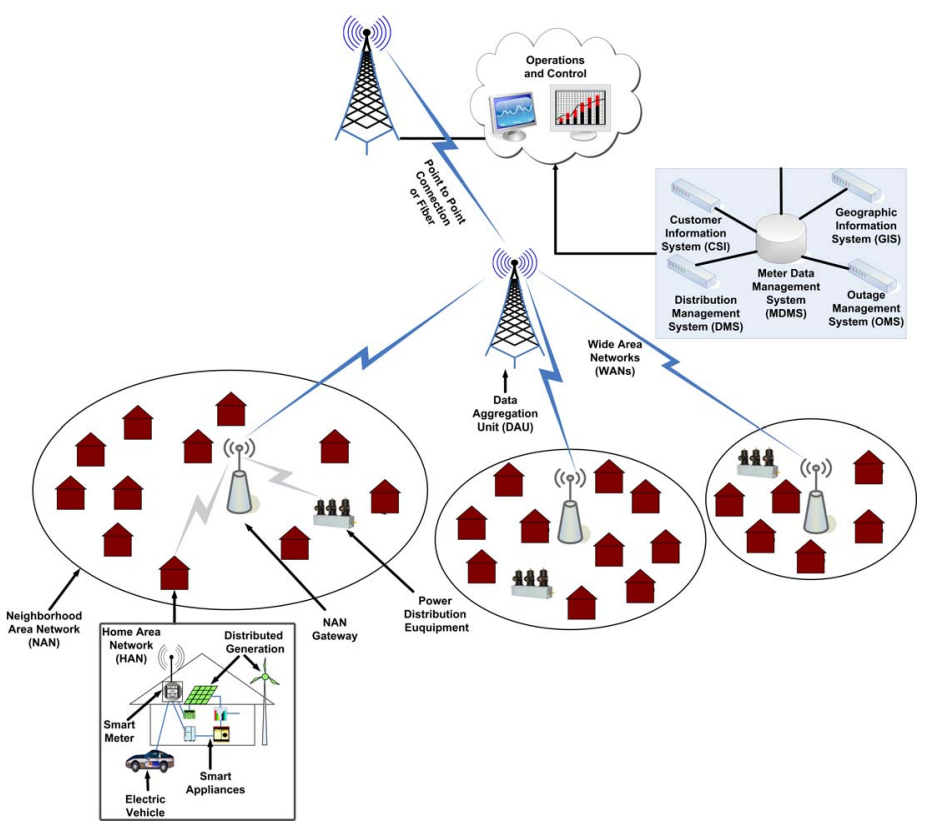

Fig. 1. The smart grid wireless network topology.

A HAN is a small-scale wireless network that connects grid components within the consumer premises such as smart appliances, smart sensors, smart meters, and energy monitoring devices. These networks provide consumers with the ability to intelligently control and monitor electrical devices within the home. The smart meter sends power information to the HAN gateway to forward meter data to the NAN. NANs connect multiple HAN gateways to transfer meter data to the data aggregation units (DAUs) via a NAN gateway. Smart grid WANs generally consist of two interconnected networks: core and backhaul. The core network connects the operations and control center with the substations, while the backhaul WANs provide broadband connectivity between NANs and the core utility systems (e.g. MDMS) via DAUs. Backhaul WANs also collect data from the distribution automation and sensor/actuator networks (for outage detection) located on the power distribution networks.

\section{Smart Grid Wireless Network Challenges}

There are several communications challenges in the hierarchical architecture of smart grid wireless networks. For NANs, the density of smart meters varies depending on the number of HANs. As a result, NANs have non-uniform demands on the network radio resources (e.g., spectrum). In addition, NAN real-time bandwidth demands can increase significantly if smart meters/sensors/actuators simultaneously transmit data in response to an event such as a power outage. Hence, some DAUs (e.g., cellular base stations) may suffer from congestion because of the variable nature of the traffic. On the other hand, communication link failures between the MDMS and smart meters can incur costs associated with power demand estimation errors and loss of consumer feedback.

The AMI requires major system improvements such as advanced distribution automation (ADA) and advanced asset 
management (AAM) for full realization of the smart grid vision. This imposes real-time constraints for transmitting load profile and power quality data. For example, data is currently collected from smart meters every 15 min, however this must be performed more frequently for full ADA support [5]. Smart meters must also transmit real-time data to the MDMS for outage management applications to prevent cascading outages and to reduce outage recovery times [5]. This can generate substantial real-time operational data, which in turn creates significant bandwidth demands on the smart grid communications infrastructure. Table I presents a classification of the data traffic for smart grid communications based on latency, packet size, and time intervals for different operation and management systems [6]. The first type of traffic includes smart meters, safety, maintenance, demand-response, billing, peak and time of use data. This is related to communications between the MDMS, OMS, CIS and the meters/sensors. The second type of traffic is related to communications between the DMS and distribution devices such as voltage regulators (VR), voltage line sensors (VLS), and phasor measurement units (PMU) [6]. These devices are attached to transformers and feeders located in the power distribution system.

\section{RAdio OVEr Fiber for SMart Grid Networks}

The arguments given in the previous section indicate that a conventional wireless architecture for smart grid communications may not satisfy the space- and time-varying traffic demands. In addition, DAU congestion is a significant possibility, and supporting the bandwidth requirements of future system extensions is a serious concern. A RoF network can be used to efficiently address these issues. This network uses optical fiber links to distribute radio frequency (RF) signals from a control unit (called the headend) to remote antenna units (RAUs). This transfers complex signal processing functions from local base stations into the headend. In this case, expensive equipment required for modulation, synchronization, multiplexing, coding, etc., can be shared among several RAUs. This greatly simplifies the RAUs, as they only need to perform opticalelectrical conversion and amplification. In addition, centralizing the signal processing facilitates the dynamic allocation of radio resources. These benefits can translate into significant system installation and operational savings.

Previous studies have considered the advantages of RoF communications (e.g., energy efficiency [4] and diversity gain [7], [8]), for conventional wireless networks. However, the smart grid wireless environment has unique communications requirements. Thus a RoF based architecture is developed in this paper to provide connectivity between smart meters, the power distribution system and the utility control centers.

RoF based WANs and NANs can support resource allocation over a range of coverage areas. In this case, the radio resources (i.e., base stations) located at the headend can be dynamically distributed to handle heterogeneous data traffic. This allows different (overlay) configurations that can provide better coverage and reduce costs while efficiently handling assignments for real-time traffic and eliminating congestion at the DAUs.

The data generated by smart meters and intelligent sensors is expected to increase significantly in the future. RoF can employ different transmission windows (e.g., optical fiber wavelengths), to provide the bandwidth necessary for current and future smart grid communications applications. It can also satisfy the QoS requirements for these applications, particularly those that are time sensitive, such as advanced distribution automation. Moreover, the RoF centralized headend can significantly reduce the signaling overhead and delays in coordinating the base stations (BSs) in conventional wireless systems.

\section{A. RoF Architecture for Smart Grid Networks}

In this section, we present the proposed RoF based clustered architecture for smart grid traffic which is illustrated in Fig. 2. A two-tier hierarchical structure is considered which includes NANs and WANs. It leverages RoF technology to facilitate efficient, flexible and reliable smart grid communications. Multiple HANs and sensors are connected to a BS located in the headend through a RAU. RAUs and BSs perform data forwarding and data aggregation similar to NAN gateways and DAUs in a conventional wireless architecture. The headend is connected to the utility control center through a fiber or high capacity point-to-point wireless connection. It would also be advantageous to combine the WANs and NANs into a larger network, since RAUs allow flexible access to various locations, and improve coverage and reliability. In this case, a NAN gateway and a DAU can be integrated to perform both data forwarding and aggregation.

The optical fiber network can be either passive or active [4]. The proposed system considers a passive network because of the lower cost and ease of deployment. Both timedivision multiplexing and wavelength-division multiplexing can be used to communicate with the RAUs. Each RAU is served by different downstream and upstream wavelengths. In the downstream direction, the data traffic is modulated at the desired radio frequency, converted to the optical domain, and transmitted to the RAUs. In the upstream direction, the RAUs receive the transmitted signals, and forward them to the headend where the desired signals are recovered for further processing [4]. The BSs can employ a number of technologies for the wireless network depending on the required coverage area, capacity and network cost. For example, WiMAX provides a large capacity with moderate coverage, LTE provides small capacity with a large coverage area, and IEEE $802.11 \mathrm{~g}$ provides medium capacity with a small coverage area [9], [10]. For illustration purposes, in this study we consider a single wireless technology, namely IEEE $802.11 \mathrm{~g}$, for all BSs.

\section{B. Efficient Cluster Based RAU Placement}

RAUs can be employed to extend the coverage area of a BS and/or increase the capacity of the RoF based WAN/NAN system. In the former case, it is envisaged that the total throughput requirements do not justify employing many BSs 


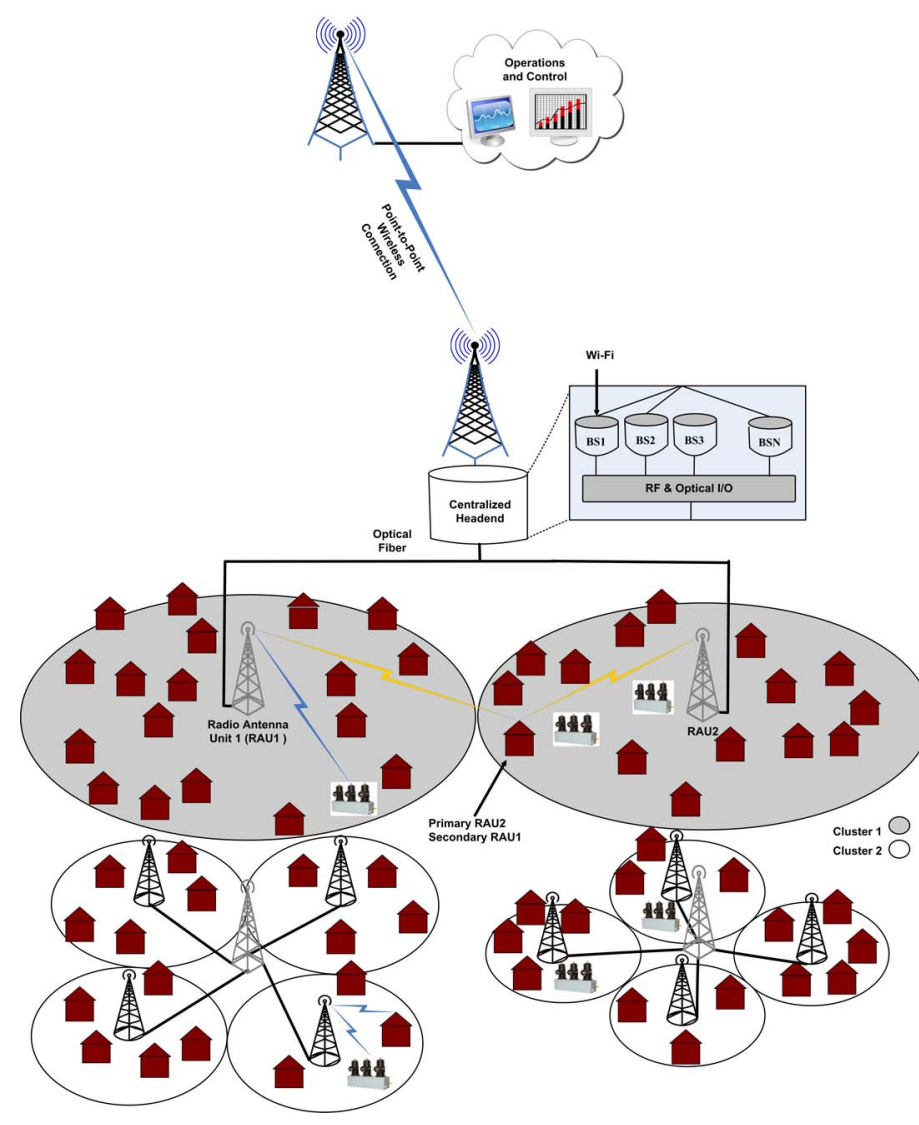

Fig. 2. The radio over fiber based smart grid network architecture.

over a coverage area. Thus, RAUs could be used in the early stages of network deployment to provide coverage over a large area at low cost (compared with using several BSs) and these distributed RAUs can be connected to a single BS [11]. In the latter case, RAUs can be used to enhance the capacity within a coverage area by providing more radio resources. This additional capacity can be used for immediate demands or the deployment of future smart grid components and applications.

RAUs should be placed based on the density of meter/sensor nodes and according to the average distance between the nodes and RAUs [12]. Each node should be within range of several RAUs to provide diversity. Previous studies [12], [13] considered minimizing the overall network cost, whereas our primary objective is efficient RAU placement to handle spatial traffic variations and improve coverage. Our approach differs from that in [13] because here meter/sensor nodes are at fixed locations, thus permitting non-overlapping regions for coverage.

A cluster is similar to a cell in that clusters can be divided into smaller ones as required. The clusters are used to determine RAU placement in a coverage area as shown in Fig. 2. The geographic area is divided into $M_{c}$ nonoverlapping regions and initially a RAU is placed at the center of each region. Assume that $\left(X\left(m_{c}\right), Y\left(m_{c}\right)\right)$ is the position of $\mathrm{RAU}_{m_{c}}, m_{c} \in\left\{1,2, \ldots, M_{c}\right\}$, which is located in cluster $c, c \in\{1,2, \ldots, C\}$. This RAU serves $N_{c}$ nodes located at positions $\left(x\left(n_{c}\right), y\left(n_{c}\right)\right), n_{c} \in\left\{1,2, \ldots, N_{c}\right\}$. The distances of all RAUs from a node are determined and placed in ascending order. The primary RAU is the one closest to the node as determined by [13]

$$
\mathrm{D}\left(\bar{m}_{c}\right)=\min _{\bar{m}_{c}}\left[\left(x\left(n_{c}\right)-X\left(\bar{m}_{c}\right)\right)^{2}-\left(y\left(n_{c}\right)-Y\left(\bar{m}_{c}\right)^{2}\right]^{1 / 2}\right. \text {. }
$$

Backup RAUs (second closest, third closest, etc.), are also determined in a similar manner. The next step is to find the set of nodes for each primary RAU (primary node), denoted by $S^{P}\left(m_{c}\right)$, and similarly the nodes for the secondary and tertiary RAUs, denoted by $S^{P+1}\left(m_{c}\right)$ and $S^{P+2}\left(m_{c}\right)$, respectively. These additional sets of nodes will be used to provide diversity through RAU cooperation. Finally, each primary RAU is optimally placed with respect to its primary nodes $S^{P}\left(m_{c}\right)$, which is the mean of the primary node locations

$$
\left(X_{\text {mean }}\left(m_{c}\right), Y_{\text {mean }}\left(m_{c}\right)\right)=\left(\sum_{n_{c}=1}^{N_{p}} x\left(n_{c}\right) / N p, \sum_{n_{c}=1}^{N_{p}} y\left(n_{c}\right) / N_{p}\right)
$$

where $N_{p}$ is the number of primary nodes for the RAU. A greedy algorithm can be used to determine the optimal placement of the clusters in each region [12]. This can be done offline and thus does not add to the computational complexity of the network. As an example, Fig. 2 illustrates typical RAU placements for the proposed clustered RoF based architecture. In this case, there are two regions for the first cluster and four regions for the second cluster. The algorithm for efficient RAU placement is given below.

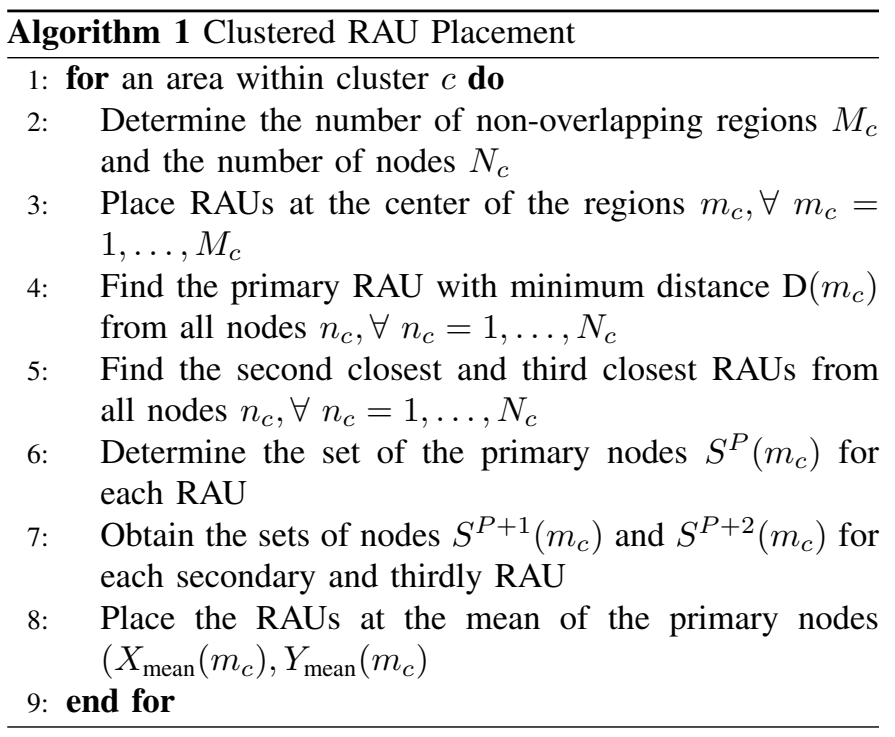

During operation, each BS can be linked to RAUs located in adjacent regions to provide transmitter and receiver diversity. Receiver diversity allows packets transmitted from a given node to be received by different RAUs. The RAU-node links will likely experience independent fading, so that diversity gains can be achieved. With knowledge of the channel state information for each RAU-node link at the headend, maximum- 
ratio combining (MRC) can be employed. RAUs in other clusters can be dynamically employed to increase this gain. With the proposed method of RAU placement, the primary nodes $S^{P+1}\left(m_{c}\right)$ can use secondary RAUs for data transmission to provide receive diversity. Figure 2 shows a node with primary RAU2 and secondary RAU1. This additional RAU can easily be selected as the secondary RAU associated with a given node has been identified. If both the primary and secondary RAUs associated with a given node are not connected to the BS at the headend, then other backup RAUs can be chosen (e.g., tertiary). This diversity can be used to eliminate coverage holes that may occur.

A node can also receive data from multiple RAUs. In this case, the BS can similarly select primary, secondary and tertiary RAUs to provide transmitter diversity. Diversity gains through cooperating RAUs were discussed in [7], however our approach differs because of the placement of RAUs in clusters.

1) Capacity planning for RAUs: As previously mentioned, full realization of the smart grid vision must meet real-time bandwidth requirements. For example, the utility control center may start a new service related to the AMI or distribution system that requires more network capacity. Further, as shown in Table I, smart grid services are dynamic and event-driven. If there is a high demand for resources associated with a given RAU, the packet error rate (PER) will increase. When the PER exceeds a threshold as defined by the required QoS of AMI services, the headend can allocate additional resources to this RAU to meet the demand. In this case, the headend can detach some of the associated nodes with a high error rate, and also allocate additional resources. Alternatively, or if there are no more channels available associated with the corresponding RAU, a portion of the load can be transferred to a new RAU (and associated BS). This provides distributed load balancing over the RAUs. Dynamic activation of RAUs and their associated BSs can be done over several clusters to lower the error probability. This approach can also be used to increase the network capacity as needed.

\section{Numerical Examples and Discussion}

This section presents numerical results which demonstrate the performance improvements possible with a RoF based network instead of conventional wireless solutions for smart grid communications. The wireless technology employed is IEEE $802.11 \mathrm{~g}$ at $2.4 \mathrm{GHz}$, however a similar investigation can be made based on other wireless technologies (e.g. IEEE802.11n or IEEE802.16x). Data traffic is considered for smart metering and the distribution system. A geographic area of $1 \times 3 \mathrm{~km}^{2}$ is divided into $2 \times 6$ regions $\left(m_{1}=12\right)$ for the first cluster $(c=1)$. The maximum radio coverage for each region is 250 $\mathrm{m}$. The optimal placement of 12 RAUs was obtained using Algorithm 1. For the second cluster, each $0.5 \times 0.5 \mathrm{~km}^{2}$ area (from the first cluster), is divided into $2 \times 2$ regions $\left(m_{2}=4\right)$. The headend is assumed to be placed in the location that minimizes the fiber cable length. The simulation parameters for the fiber optic system are given in Table II. The optical link budget calculation based on a single-mode fiber network shows negligible optical signal loss for the maximum fiber cable length of $2.5 \mathrm{~km}$.

To obtain non-uniformly distributed traffic over the coverage area, different meter densities are considered for the regions in the first cluster, but the traffic is uniformly distributed within each region. The meter density varies between 1000 and 2000 per $\mathrm{km}^{2}$. The maximum density corresponds to an urban area as reported in [14]. We also consider one polemounted transformer for every 7 customers. The number of nodes for each grid component are given in Table II. For the area under study, we determined that the minimum bandwidth requirements for downlink and uplink traffic are $5 \mathrm{Mbps}$ and $11 \mathrm{Mbps}$, respectively. The uplink traffic includes data traffic related to two cameras located in a substation. Both the uplink and downlink consist of $20 \%$ overhead traffic.

1) Outage probability: We examine the effect of diversity gains provided by the clusters in the coverage area. The physical (PHY) layer radio parameters from [14] are given in Table II. The uplink and downlink in the RoF based network operate at $12 \mathrm{Mbps}$ and $6 \mathrm{Mbps}$, respectively. We also consider the outage probability for the conventional wireless network as a basis for comparison with the RoF based WAN/NAN system. The wireless propagation model given in [14] is employed with shadowing and Rayleigh fading. For adjacent regions within a cluster, the two RAUs are connected to one BS located in the headend. In the absence of diversity, the BS communicates with the nodes through the primary RAUs. If diversity is employed, a node communicates with the BS through its primary and secondary or tertiary RAUs. We also consider two non-overlapping channels based on the IEEE $802.11 \mathrm{~g}$ standard.

We first consider receive diversity where MRC is used at the headend for the uplink signals. In this case, two RAUs operating at different frequencies receive signals from a node. Figure 3 shows the average outage probability over all regions as a function of the maximum coverage radius, and the average outage probability for clusters with and without receive diversity. From this figure, performance improvements are evident in terms of the outage probability when clustering with and without diversity is employed. The coverage radius corresponding to a $0.1 \%$ outage probability is $250 \mathrm{~m}$ and $192 \mathrm{~m}$ for the second cluster with and without diversity, respectively. The maximum coverage is significantly reduced from $120 \mathrm{~m}$ with diversity to $70 \mathrm{~m}$ without diversity for the first cluster.

The PHY data rate on the downlink is $6 \mathrm{Mbps}$. In this case, the coverage radius without diversity corresponding to a $0.1 \%$ outage probability is approximately $185 \mathrm{~m}$ and $245 \mathrm{~m}$ for the first and second clusters, respectively. A greater coverage radius can be achieved with transmitter diversity. As stated in [7], signal phases can be shifted for different RAUs and the transmission power optimally allocated across the RAUs. The conventional wireless network has a data rate of $1 \mathrm{Mbps}$ in both the uplink and downlink at a distance of $250 \mathrm{~m}$ for a $0.1 \%$ outage probability. The outage probability for uplink and downlink data rates of 12 and $6 \mathrm{Mbps}$ is also shown. Note that 
this network only has a radio system with BSs. Conversely, the combined RoF based clustered system with diversity can provide sufficient coverage for a WAN/NAN of $1 \times 3 \mathrm{~km}^{2}$ with a $0.1 \%$ outage probability. This provides data rates of 12 and $6 \mathrm{Mbps}$ for the uplink and downlink, respectively.

2) Traffic latency: Several traffic types from Table I are considered. All data streams are constant bit rate (CBR) traffic and TCP connections are used for all data traffic. Retransmissions are allowed if a packet is corrupted. There is also data traffic related to video surveillance in distribution substations. Packets are generated every $5 \mathrm{~ms}$ with a packet length of 1000 bytes. To evaluate the performance of the proposed RoF based WAN/NAN network, we consider the average latency of all packets of the same traffic type. The latency of a packet is defined as the time from when the packet is buffered at the source node to the time the packet arrives at the destination node.

The IEEE 802.11 MAC layer is implemented using the NS2.33 simulator as described in [4]. The simulation time was $7.2 \times 10^{3} \mathrm{~s}$. For an average outage probability of $0.1 \%$, Fig. 3 shows that with the proposed technique the second cluster covers the entire area for both the uplink at $12 \mathrm{Mbps}$ and the downlink at $6 \mathrm{Mbps}$. For the same radio coverage area and outage probability, the conventional wireless network has a data rate of only $1 \mathrm{Mbps}$ for both the uplink and downlink. Figure 4 shows the latency for several data traffic types using conventional wireless communications and the proposed approach. The conventional system has large transmission delays in both the uplink and downlink for the majority of traffic types. However, the proposed clustered technique can ensure the low latency needed for smart grid traffic while providing a wide coverage area with a low outage probability of $0.1 \%$.

\section{CONCLUSION}

In this paper, the application of radio over fiber (RoF) for smart grid communications was introduced. The potential of RoF technology in smart grid communications and its benefits were identified. A clustered architecture was proposed for strategic RAU placement to handle spatial traffic variations and improve radio coverage. It was shown that transmission and reception diversity gains can be facilitated by using clusters. This can increase the capacity and improve the coverage of smart grid wireless networks. Numerical results were presented which show that a significant performance improvement can be achieved in terms of latency and coverage area using the proposed method compared to a conventional wireless system.

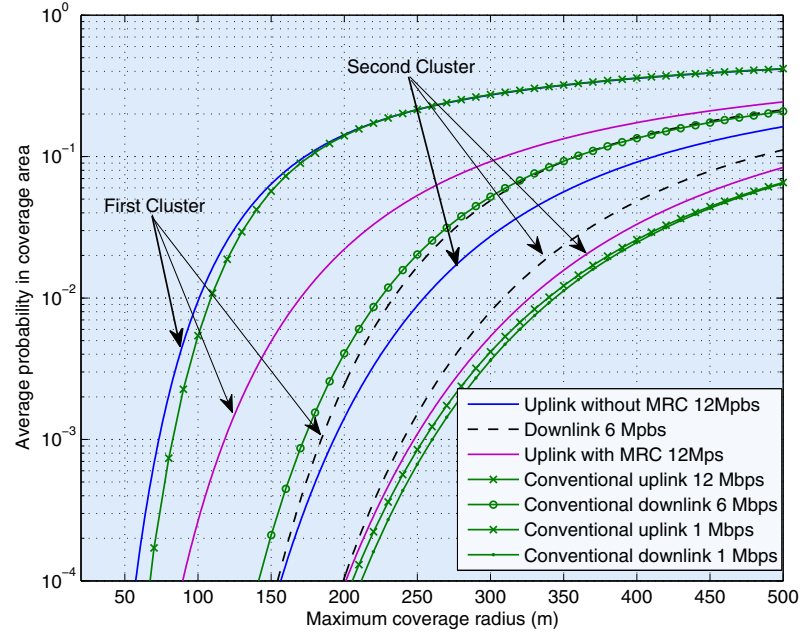

Fig. 3. Outage probability for the coverage area based on IEEE $802.11 \mathrm{~g}$ technology at $2.4 \mathrm{GHz}$.

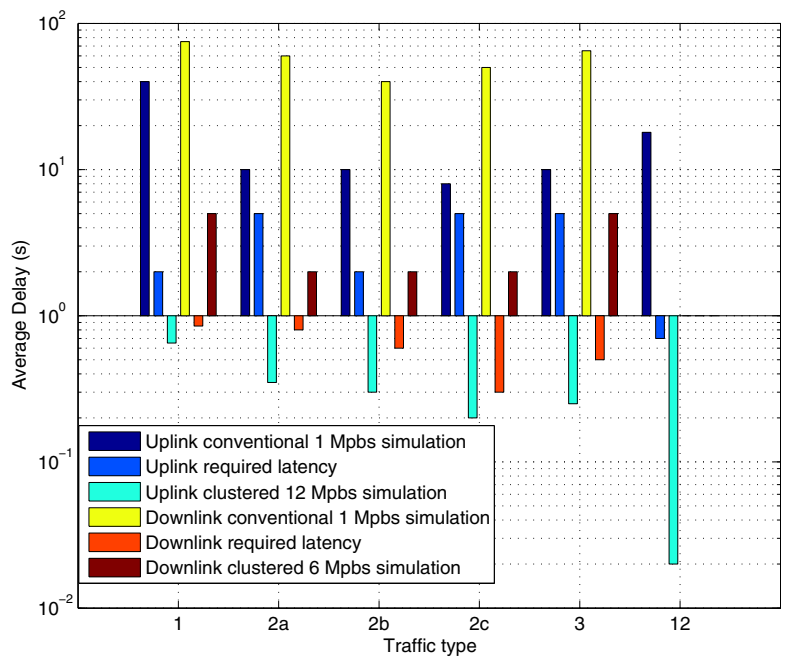

Fig. 4. Latency for the proposed clustered approach and conventional method for several types of data traffic from smart meters and the distribution system.

\section{REFERENCES}

[1] H. Farhangi, "The path of the smart grid," IEEE Power and Energy Mag., vol. 8, no. 1, pp. 18-28, Jan.-Feb., 2010.

[2] A. Ghassemi, S. Bavarian, and L. Lampe "Cognitive radio for smart grid communications," in IEEE Int. Conf. on Smart Grid Commun., Gaithersburg, MD, pp. 297-302, Oct. 2010.

[3] V. C. Gungor and D. Sahin, "Cognitive radio networks for smart grid applications: A promising technology to overcome spectrum inefficiency," IEEE Veh. Tech. Mag., vol. 7, no. 2, pp. 41-46, June 2012.

[4] A. L. Haoming, A. Attar, and V. C. M. Leung, "Collision avoidance and mitigation in cognitive wireless local area network over fibre," in Proc. Int. Conf. Evolving Internet, Cannes/La Bocca, France, pp. 133-138, Aug. 2009.

[5] US Dept. of Energy, Office of Electricity Delivery and Energy Reliability, Advanced Metering Infrastructure, 2008.

[6] R. Cunningham et al., SG Network System Requirements Specifications, Interim Release 3, US Dept. of Energy, 2010. 
TABLE I

DATa TRAFFiC Classification FOR SMART GRid COMMUNiCATIONS [6]

\begin{tabular}{|c|c|c|c|c|c|}
\hline Type & $\begin{array}{c}\text { DMS } \\
\text { Control and Monitoring }\end{array}$ & Device & $\begin{array}{l}\text { Payload Size (bytes) } \\
\text { Uplink, Downlink }\end{array}$ & $\begin{array}{c}\text { Interval } \\
\text { Uplink, Downlink }\end{array}$ & $\begin{array}{c}\text { Latency (s) } \\
\text { Uplink, Downlink }\end{array}$ \\
\hline 1 & Power Quality & PMU & 1536,150 & $5 \min , 5 \min$ & $<5,<2$ \\
\hline 2 & $\begin{array}{l}\text { Local Substations } \\
\text { Line Loads, Voltage }\end{array}$ & Feeder VR, VLS & $\begin{array}{l}\text { a. } 50,250 \\
\text { b. } 1000,250 \\
\text { c. } 1000,150\end{array}$ & $\begin{array}{c}5 \mathrm{~min}, 5 \mathrm{~min} \\
15 \mathrm{~min}, 5 \mathrm{~min} \\
60 \mathrm{~min}, 15 \mathrm{~min}\end{array}$ & $\begin{array}{l}<5,<2 \\
<2,<2 \\
<5,<2\end{array}$ \\
\hline 3 & Distribution Transformers & Transformer & 250,150 & $5 \mathrm{~min}$ and $5 \mathrm{~min}$ & $<5$ and $<5$ \\
\hline 4 & $\begin{array}{l}\text { Measurement Equipment, } \\
\text { Component Temperatures }\end{array}$ & Sensor & $\geq 64, \geq 64$ & event-driven & $<2,<2$ \\
\hline 5 & Circuit Breaker Status & Sensor & $\geq 64, \geq 64$ & event-driven & $<2,<2$ \\
\hline 6 & Distributed Generators & Feeder Sensor & 250,150 & $5 \mathrm{~s}, 5 \mathrm{~s}$ & $<2,<2$ \\
\hline & OMS, CIS, MDMS & Device & Payload Size (bytes) & Interval & Latency (s) \\
\hline 7 & Outage Detection & Sensor and Meter & $200-2400$ & event-driven & $<5$ \\
\hline 8 & Theft Detection & Sensor and Meter & $200-2400$ & event-driven & $<5$ \\
\hline 9 & $\begin{array}{l}\text { Energy Consumption, } \\
\text { Customer Generators }\end{array}$ & Smart Meter & $200-2400$ & Periodically & $<15$ \\
\hline 10 & $\begin{array}{l}\text { Time of use Rates } \\
\text { and Peak Hours }\end{array}$ & Smart Meter & $200-2400$ & $30-60 \mathrm{~min}$ & $<5$ \\
\hline 11 & Demand Response & Smart Meter & $200-2400$ & Periodically & $<5$ \\
\hline 12 & $\begin{array}{l}\text { Meter Reading } \\
\text { and Services }\end{array}$ & Smart Meter & $200-2400$ & 25 per day for 1000 meters & $<15$ \\
\hline & Other Traffic & Device & Bandwidth (Mbps) & Latency (s) & \\
\hline 13 & $\begin{array}{c}\text { Video Surveillance: } \\
\text { Distribution Substations }\end{array}$ & $\begin{array}{c}\text { Camera, Video } \\
\text { Compression MPEG4 }\end{array}$ & 2 & $<0.02$ & \\
\hline
\end{tabular}

TABLE II

SIMULATION PARAMETERS

\begin{tabular}{|c|c|c|c|c|c|}
\hline \multicolumn{2}{|l|}{ Power Distribution System } & \multicolumn{2}{|l|}{ Radio System } & \multicolumn{2}{|l|}{ Fiber Optic } \\
\hline Node & Quantity & PHY Model Parameter & Value & Single Mode Parameter & Value \\
\hline Smart meter & 3528 & RAU Antenna Gain (dBi) & 12 & $\begin{array}{c}\text { Fiber Cable Loss } \\
(2.5 \mathrm{~km}, \mathrm{~dB})\end{array}$ & 0.9 \\
\hline $\begin{array}{l}\text { Feeder Low Voltage } \\
\text { Line Sensor }\end{array}$ & 3528 & Receiver Antenna Gain (dBi) & 12 & $\begin{array}{l}\text { Connector, Splitter } \\
\text { Switch Loss (dB) }\end{array}$ & 48 \\
\hline Transformer & 504 & $\begin{array}{l}\text { PHY Data Rate (Mbps) } \\
\text { Uplink, Downlink }\end{array}$ & 12,6 & $\begin{array}{l}\text { Passive Device } \\
\text { Attenuation }(\mathrm{dB})\end{array}$ & 3 \\
\hline $\begin{array}{l}\text { Feeder Medium Voltage } \\
\text { Line Sensor }\end{array}$ & 504 & $\begin{array}{l}\text { Required SNR (dB) } \\
\text { Uplink, Downlink }\end{array}$ & 11,8 & Receiver Sensitivity (dBm) & -30 \\
\hline Phase Measurement Unit & 3884 & $\begin{array}{l}\text { Receiver Sensitivity }(\mathrm{dBm}) \\
\text { Uplink, Downlink }\end{array}$ & $-86,-90$ & $\begin{array}{l}\text { Transmit Power }(\mathrm{dBm}) \\
\text { Transmit Power }(\mathrm{dBm})\end{array}$ & $\begin{array}{l}0 \\
0\end{array}$ \\
\hline Feeder Voltage Regulator & 3883 & Radio Transmit Power (dBm) & 20 & Optical Link Budget (dB) & -0.4 \\
\hline Substation Camera & 2 & Implementation Loss (dB) & 3 & & \\
\hline Substation & 1 & & & & \\
\hline
\end{tabular}

[7] A. Attar, A. L. Haoming, Q. Pang, and V. C. M. Leung, "Cognitive wireless local area network over fibers: Architecture, research issues and testbed implementation," IEEE Commun. Mag., vol. 50, no. 6, pp. 107-113, June 2012.

[8] L. Haoming, J. Hajipour, A. Attar, and V. C. M. Leung, "Efficient HetNet implementation using broadband wireless access with fiber-connected massively distributed antennas architecture," IEEE Wireless Commun., vol. 18, no. 3, pp. 72-78, June 2011.

[9] F. Diehm et al., "The FUTON prototype: Broadband communication through coordinated multi-point using a novel integrated optical/wireless architecture," in Proc. IEEE Globecom Commun. Conf., Miami, FL, pp. 757-762, Dec. 2010.
[10] The FUTON project website: www.ict-FUTON.eu.

[11] N. J. Gomes, P. P. Monteiro, and A. Gameiro, Next Generation Wireless Communications Using Radio over Fiber, Wiley, New York, 2012.

[12] S. Sarkarl, B. Mukherjeel, and S. Dixit, "Optimum placement of multiple optical network units (ONUs) in optical-wireless hybrid access networks," in Proc. IEEE Optical Fiber Commun. Conf., Anaheim, CA, pp. 1-3, Mar. 2006.

[13] P. H. Gomes, N. L. S. da Fonseca, and O. C. Branquinho, "Bi-criteria optimization of radio resources for radio-over-fiber access networks," in Proc. IEEE Int. Conf. Commun., Kyoto, Japan, pp. 1-6, June 2011.

[14] NIST Priority Action Plan 2, Guidelines for Assessing Wireless Standards for Smart Grid Applications, ver. 1.0, Dec. 2010. 